\title{
Assessment of adolescents' diet at 14 years in the Danish National Birth Cohort: Development of questionnaire and perspectives for research
}

\author{
Sjurdur Olsen ${ }^{1,2}$ and Anne Bjerregaard ${ }^{1}$ \\ ${ }^{1}$ Statens Serum Institut, Copenhagen, Denmark and \\ ${ }^{2}$ Department of Nutrition, Harvard TH Chan School of Public Health, Boston, USA
}

\section{Abstract}

Introduction: By October 2002, a little more than 100,000 women in their early pregnancies had been recruited in the Danish National Birth Cohort (DNBC), for long-term follow-up of themselves and their offspring. The overall purpose of DNBC is the establishment of a research database comprising information on exposures and other factors during pregnancy and early life that can be linked to health-related phenomena occurring throughout life. More than 90,000 live-born children are followed by questionnaires and registry linkages, and more than 500 peer-reviewed articles have so far been published from DNBC.

The latest completed assessment in the children recorded diet and physical activity at age 14. Our group was responsible for the development and implementation of the questionnaire in DNBC. We report on its development; on selected data; and on their potential utility for research.

Materials and methods: The questionnaire was an on-line food-frequency questionnaire (FFQ) comprising 158 food items. It was a substantially modified version of the questionnaire developed for the offspring of Nurses' Health Study 2 participants (GUTS) adapted to the Danish food culture.

Results: Mean BMI was 19, and $9.1 \%$ of boys and $8.3 \%$ of girls were overweight ( $\mathrm{n}=$ the first 20,814 completed questionnaire). For boys and girls, respectively, estimated mean (SD) intakes were: Energy 9.3 (3.5) and 10.4 (3.9) MJ/d; Protein 15.7 (2.4) and 15.8 (2.5) E\%; Fat 32.1 (4.6) and 32.6 (4.5) E\%; Carbohydrate 52.2 (5.3) and 51.5 (5.3) E\%; Sugar 7.8 (4.1) and 7.1 (3.8) E\%; Fruits 213 (208) and 253 (222) g/d; Vegetables 201 (142) and 240 (163) g/d; Fish 96 (108) and 94 (107) g/w; Meat 792 (415) and 694 (381) g/week; and Sugar-sweetened beverages $1.2(1.3)$ and $0.8(1.1) \mathrm{L} /$ week.

Considerable variability was observed. E.g., for sugar-sweetened beverages, the highest quarter consumed more than 1.4 and the lowest less than $0.32 \mathrm{~L} /$ week; for fish, the corresponding figures were 120 and $30 \mathrm{~g} / \mathrm{week}$, respectively. We observed substantial gradients across sociodemographic groups.

Discussion: Our data revealed a considerable variability in dietary intake in DNBC, with substantial sociodemographic gradients. These results point to an important and unique potential for prevention and future research. DNBC is the largest database worldwide of its kind with extensive information on dietary exposures in pregnancy (1) as well as during adolescence.

\section{Funding}

Innovation Fund Denmark and Nordea-fonden.

\section{Conflict of Interest}

There is no conflict of interest

\section{Reference}

1. Olsen SF et al. (2007) Paediatr Perinat Epidemiol PMID:17239183. 